\title{
A LASER SYSTEM FOR THE CONTROL OF THE COMPLEX FOR THE CONSTRUCTION OF MINI
}

\author{
Alexey Grigoryevich Bulgakov ${ }^{1^{*}}$ Alexander Vakolyuk ${ }^{2}$ Nikolay Glebov² Natalia Bienkowski \\ ${ }^{1}$ Southwest State University, Kursk, Russia \\ ${ }^{2}$ South-Russian State Polytechnic University, Novocherkassk, Russia \\ ${ }^{3}$ Technical University Dresden, Germany
}

This article describes a laser system for the control and controlling of mini shields in a tight urban development. The direction of movement of the mini shield at the start point is set with the aid of a beam generated by the source of laser radiation. Using the same source is transferred to the service information from the operator's console for the equipment mini shield. On mini shield installed equipment defining two points mini shield relative to the laser beam, as well as take on the connection information. Information on the operator panel are transmitted using a second laser emitter mounted on a mini shield.

Key words: Robotics and mechatronics, Automation and control, Tunneling complex, Laser system

\section{INTRODUCTION}

Municipal services of cities-megacities in different countries more and more attention is paid to the use of advanced technologies trenchless recovery (rehabilitation) and construction of new water supply, drainage and other utilities, which is an alternative to traditional open method of reconstruction and construction of pipelines of excavation and trenching methods $[01,02]$. To date, the most promising equipment for this type of work are different tunneling and mini shield complexes, generally representing robotic and mechatronic systems.

A significant part of all underground utilities are installed in boreholes with a diameter up to $1000 \mathrm{~mm}$, which does not allow operators and maintenance staff to be in the small board, thereby imposing specific requirements for control and management. Based on this development task of the principles, methods and tools to increase the efficiency, flexibility and safety of operation of mechatronic systems for the construction of mini tunnels it is very important.

It was found that to improve the efficiency and safety of operation of tunnel boring equipment must be equipped with specialized systems controlling the spatial position of the mini shield, state of technological equipment and the exchange of information between the remote operator systems and equipment mini shield. On the basis of this analysis of existing control systems tunnel boring complexes of medium and large diameters revealed that most are optimal laser control system [03,04,05].

\section{A MATHEMATICAL MODEL OF THE MECHATRONIC TUNNEL-BORING COMPLEX}

To determine the spatial position of mechatronic tunnel-boring complex on the base of mini shield relative to the base direction and doing it in a given direction is sufficient to control the coordinates of the two points of the longitudinal axis of the mini shield or the coordinates of one point of the axis and the angles between the axis of the mini shield and its projections on the coordinate plane [06]. Taking into account the technological and design features of the mini shields, not always possible to determine the coordinates of theirs two point longitudinal axis and its position is necessary to determine the coordinates of two points are not on the axis of the mini shield. Figure 1 shows a mini shield of circular cross section of diameter $\mathrm{d}$ and length $l_{M}$ in several coordinate systems with randomly selected points $D$ and $E$ : OXYZ - base coordinate system, located on the Earth's surface (datum); $\mathrm{O}_{\mathrm{P}} \mathrm{X}_{\mathrm{P}} \mathrm{Y}_{\mathrm{P}} \mathrm{Z}_{\mathrm{P}}$ - coordinate system related to the project axis traversed by small tunnel; $O_{L} X_{L} Y_{L} Z_{L}$ - coordinate system associated with the laser setter direction and relative to it a determination of $D$ and $E$ of point coordinates; $\mathrm{O}_{M} \mathrm{X}_{M} \mathrm{Y}_{M} Z_{M}-$ coordinate system associated with the point of the tail shield mini.

For the successful conduct of the mini shield on a given project path you need to know the coordinates of the $\mathrm{H}$ point relative to the reference coordinate system and the angles between the axis of mini shield and its projections on the coordinate plane. The coordinates of the $\mathrm{H}$ pointmay be determined by the following expression:

$$
\begin{gathered}
X_{H}=l_{M} \cdot \cos \alpha \cdot \cos \beta+\sqrt{L^{2}-Y_{\mathrm{oM}}{ }^{2}-Z_{\mathrm{oM}}{ }^{2}} \\
Y_{H}=l_{M} \cdot \cos \alpha \cdot \sin \beta+Y_{\mathrm{oM}} \\
Z_{H}=-\left(l_{M} \cdot \sin \alpha+Z_{\mathrm{oM}}\right)
\end{gathered}
$$

where $L$ - traveled path of mini shield, measured from the beginning of $\mathrm{O}_{\mathrm{P}} \mathrm{X}_{\mathrm{P}} \mathrm{Y}_{\mathrm{P}} \mathrm{Z}_{\mathrm{P}}$ coordinate system; $\mathrm{Y}_{\mathrm{OM}}, \mathrm{Z}_{\mathrm{OM}}$ - coordinates of point $\mathrm{O}_{\mathrm{M}}$ in the basic coordinate system.

In the above expressions are four unknown quantities: $\alpha, \beta, Y_{\mathrm{OM}}, Z_{\mathrm{OM}}$. 
Given the angle of mini shield heel $\varphi_{\mathrm{K}}$, the tilt angle $\alpha$ can be determined from the following relationship:

$$
\operatorname{tg} \alpha=\left[\left(z_{L E}-z_{L D}\right) \cdot \cos \varphi_{\mathrm{K}}+\left(y_{L D}-y_{L E}\right) \cdot \sin \varphi_{\mathrm{K}}\right] / b_{2}
$$

wherey $_{\mathrm{LE}}, \mathrm{Z}_{\mathrm{LE}}, \mathrm{y}_{\mathrm{LD}}, \mathrm{Z}_{\mathrm{LD}}-$ coordinates of points $D, E$ in projections on the axis $Y_{L} и Z_{L}$ coordinate system associated with the laser setter direction (known coordinates).

Course angle $\beta$ is determined by the expression:

$$
\operatorname{tg} \beta=\left[\left(z_{L E}-z_{L D}\right) \cdot \sin \varphi_{\mathrm{K}}+\left(y_{L E}-y_{L D}\right) \cdot \cos \varphi_{\mathrm{K}}\right] / b_{2}
$$

The coordinates of $\mathrm{O}_{M}$ can be identified by the expressions of the following for:

$$
\begin{aligned}
Y_{\mathrm{OM}}=\Delta Y_{L}-\left[h \cdot \sin \varphi+z_{L D}\right. & \left.\cdot \sin \varphi_{\mathrm{K}}+y_{L D} \cdot \cos \varphi_{\mathrm{K}}+b_{1} \cdot \operatorname{tg} \beta\right] \\
& \cdot \cos \beta \\
Z_{\mathrm{OM}}=\Delta Z_{P}-\Delta Z_{L}+\left[h \cdot \cos \varphi+z_{L D} \cdot \cos \varphi_{\mathrm{K}}-y_{L D} \cdot \sin \varphi_{\mathrm{K}}-b_{1} \cdot \operatorname{tg} \alpha\right] & \cdot \cos \alpha
\end{aligned}
$$

where $\varphi=\varphi_{\mathrm{H}}+\varphi_{\mathrm{K}}, \varphi_{\mathrm{K}}-$ minibus roll angle; $\varphi_{\mathrm{H}}$ initial angle.

Thus, it can be concluded that, to determinet hespatial coordinates in the mini shield coordinates OXYZ reference system is necessary to determine the coordinates of two points to another mini shield $\mathrm{O}_{\Omega} \mathrm{X}_{\Omega} \mathrm{Y}_{\Omega} \mathrm{Z}_{\Omega}$ coordinate system with respect to which there is the possibility of a direct measurement by various technical means.

Based on the analysis developed a universal functional system diagram (Figure 2), which allows for the definition of the spatial coordinates of the two points of the mini shield relative to the beam of the laser set point, as well as the exchange of information between the control panel and equipment mini by two laser beams [07].

It was determined that the construction of tunnels with large and small diameters is carried out using a large number of processing machines and units, as well as control systems. For effective and quality construction of tunnels all equipment, including a system for determining the spatial coordinates of a mini shield and exchange of information, should function for a particular algorithm. The system task is to monitor the situation regarding the mini shield design direction, the poll gauges the state of the process equipment, advanced data processing and exchange of information with a computer operator for the laser channels of information transmission.

From a functional system developed scheme shows that it consists of two modules, designed for installation in the starting pit (equipment operator) and mini shield (hardware of mini shield), each module should operate in their particular algorithm. To perform the tasks assigned to the system, it should function in the following sequence: to scan photodetector arrays with subsequent calculation of the coordinates of the center of the laser beam; interrogate the sensors of the process equipment and to carry out primary processing of the information received; to carry out information exchange session with the operator's computer through an optical channel; decode and execute commands received from the computer operator.

On the basis of the functional diagram of the system determining spatial coordinates mini shield and information exchange, as well as the algorithm of functioning of its modules, performed computer simulations and study of this system in order to check its performance, positioning accuracy and other parameters.

System study is divided into several stages:

- study of accuracy of positioning the center of the laser beam depending on the step in the matrices of photodetectors and beam diameter;

- study the noise immunity of the system to the ambient light photosensor optical radiation of different frequencies and capacity;

- validation of data transmitted in the operator's computer.

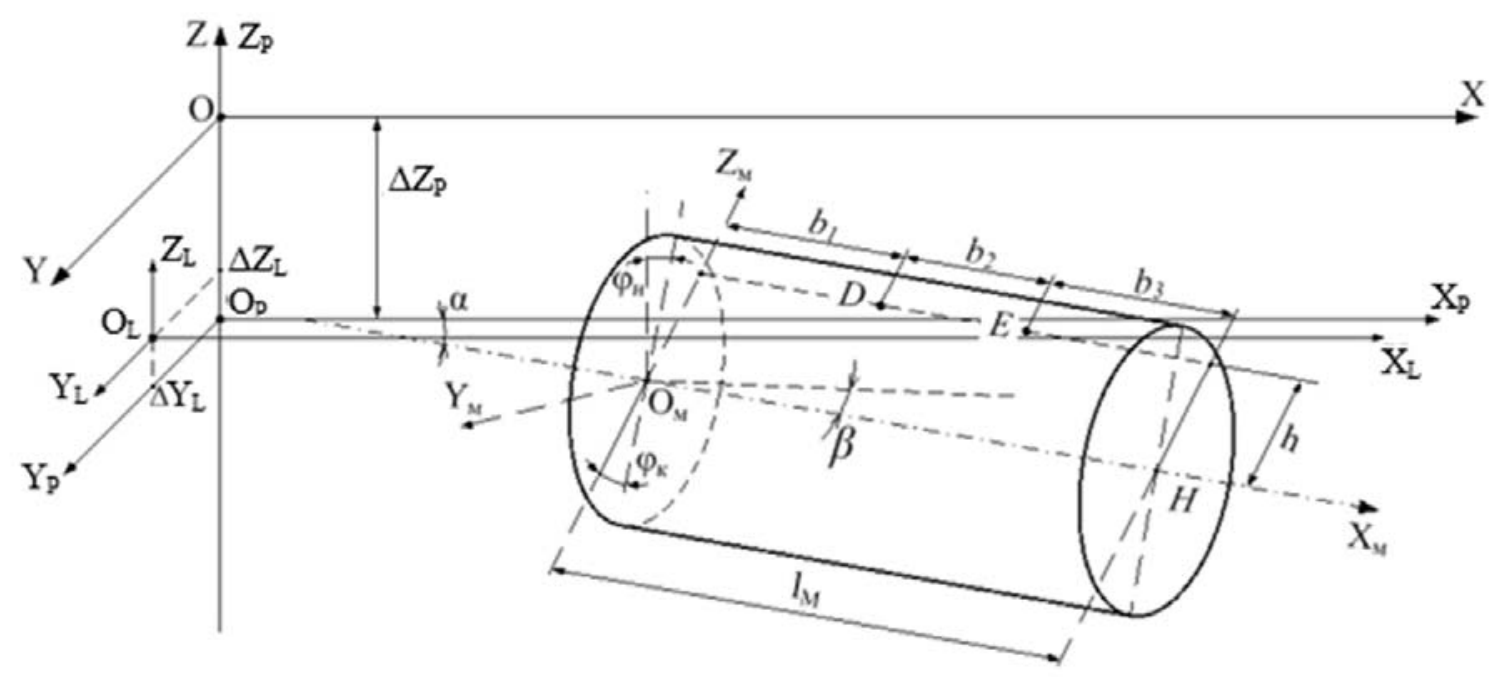

Figure 1: Mini shield in several coordinate systems 
Operating environment

Shields hardware

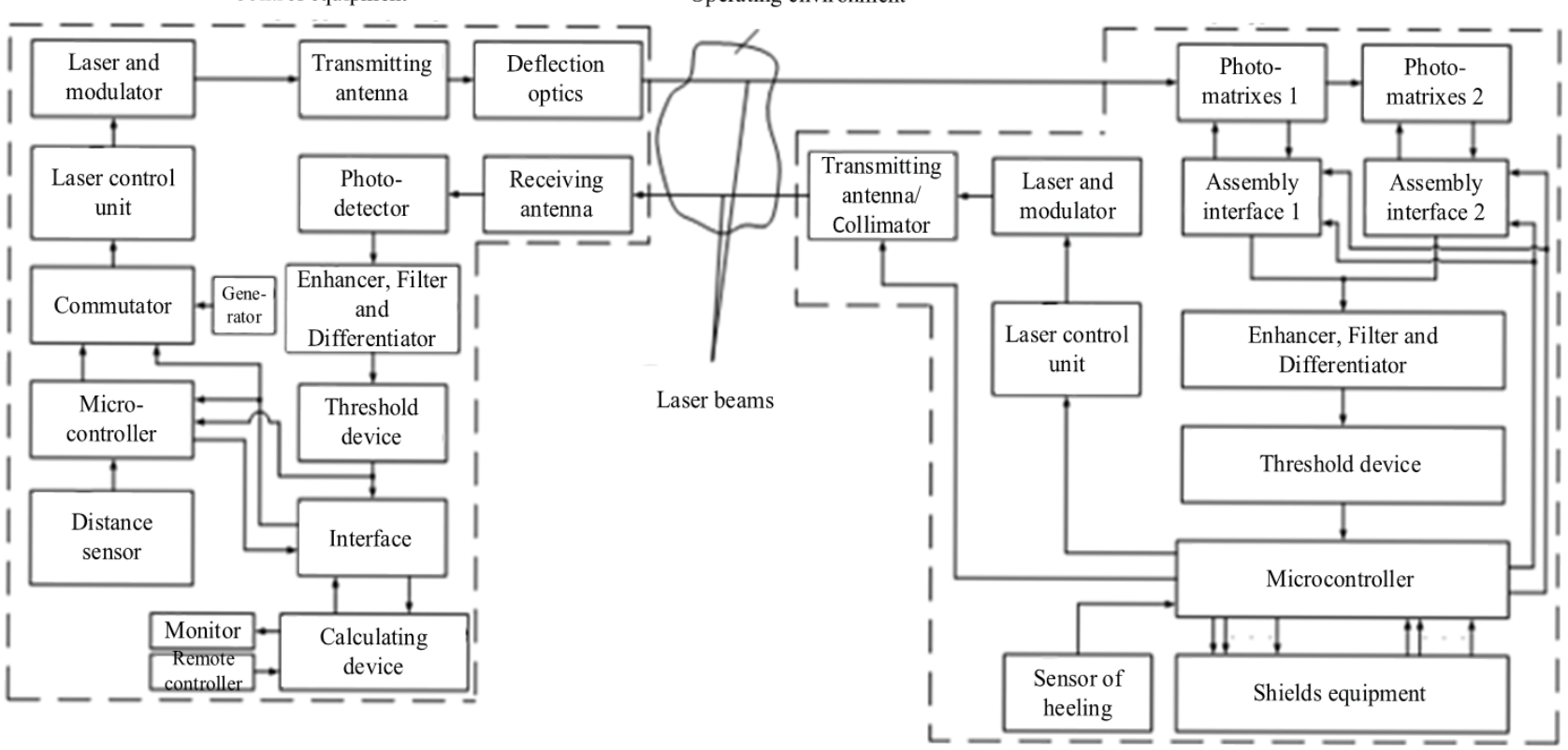

Figure 2: Functional diagram of a system determining the spatial coordinatesmini shield complex and information-sharing

It was established that for stable determining spatial coordinates of mechatronic mini shield with accuracy \pm 5 $\mathrm{mm}$ can be recommended design step photodetector matrices with elements of $10 \mathrm{~mm}$ and a diameter of the laser beam direction set point 17-20 mm.

To investigate the system noise immunity to ambient light, the photodetector together with the useful signal fed to the noise signals representing sinusoidal signals of varying amplitude and frequency.

Developing of effective opto-mechanical control and motion controlling devices of mechatronic mini shield for the construction of mini tunnels, operating in atmospheric conditions of mini tunnel, is impossible without taking into account its influence on the properties of laser light. The main effect of the atmosphere is reduced to decrease the optical power, recorded at a distance from the source, due to the effects of scattering and absorption in the solid and liquid particles in it are suspended. As a result, the total transmittance studies at various initial data obtained graphs showing that the power of the laser radiation, recorded at a distance from the radiation source, decreases exponentially as the distance from it, and the intensity of the power reduction depends on the meteorological visibility range.

Based on the previously developed algorithms of the system operation, used in its modeling, of selected components and circuit diagrams, synthesized algorithms of the physical functioning of the sample, developed the control software included in the devise of microcontrollerand built an experimental model system (Figure 3).
Laboratory testing of the product showed that it meets all the requirements put forward by: determines the coordinates of the center of the laser beam with an accuracy of $\pm 5 \mathrm{~mm}$, has a high immunity to ambient light, communicates with a computer operator at a rate of $C=115.2 \mathrm{kbit}$ / s and operates stably at a distance up to $1 \mathrm{~km}$.

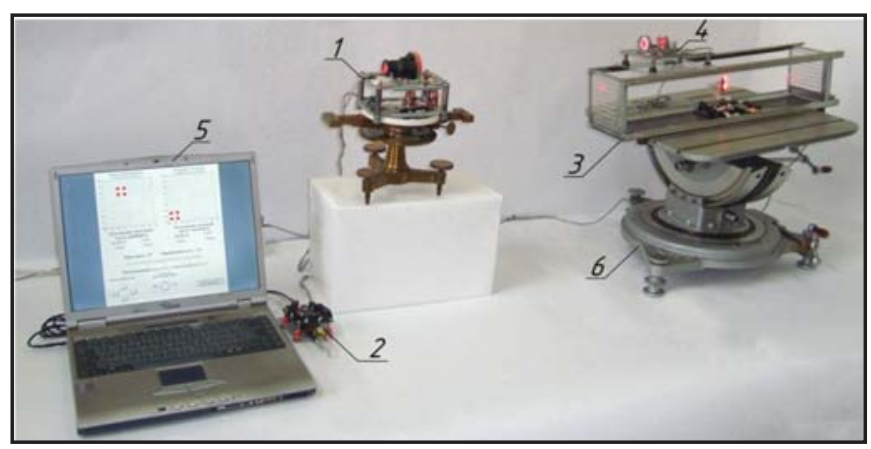

Figure 3: Stand for laboratory testing determination system spatial coordinates of mini shield and exchange of information: 1 - module laser receiver-transmitter operator; 2 - the operator information processing module;

3 - module positioning of mini shield;

4 - laser reverse channel transmitter;

5 - computer of operator; 6 - directional table 


\section{CONCLUSIONS}

The studies revealed that the developed system allows determination of the spatial coordinates of the moving object with the required accuracy and reliable exchange of information with the equipment installed on them. This allows remote interactive controlling of mobile equipment in the construction of tunnels and other underground structures for various purposes. The device can be used both in the construction of tunnels of small, large diameter tunnels and for controlling terrestrial mobile units. In addition, the device can be used as the stationary optical information exchange line.

\section{REFERENCES}

1. Bulgakov A., Vorobyov V., Evtushenko S., Parshin D. Automation and robotic in construction. - Moscow: INFRA-M, 2013. - 452 p.

2. KlorikyanV., HodoshV. Tunnelling machines and complexes. - Moscow: Publishing Bowels, 1977. $326 \mathrm{p}$.

3. Lasersystemforcontrolthemovingdirectionoftunnelling machines/ N. Glebov, V. Zagorodnyuk, N. Kruglov // Mining machinery and automation: Proceeding / YNIIPcoal. -Moscow, 1974. -Nr. 4(169). - pp. 8-10.

4. Patent 2206751 RF, MPK 7E21Д9/093, E21C35/24. An automatic control system for the movement of machinery and the construction of the shield lining of tunnel complex/ N. Glebov, Y. Miroshnikov, S. Pritchin.- Nr. 2001128576/03; publ. 20.06.2003, bul. Nr.17.
5. GlebovN., PritchinS. Acontrollingsystemthe moving of mechatronic tunneling complex // Mechatronic. 2003. - Nr 8. - pp. 19-23.

6. Glebov N.Controllingsystemsofmechatroniccomplexesinunderground and industry construction/ $\mathrm{N}$. Glebov // News of TRTU / Radio Engineering University Taganrog: Publishing TRTU, 2006. - Nr 3, pp. 85-92.

7. Patent 2405937 RF, MPK E21D 9/093, E21C 35/24. A system for control and controlling the moving of mini shield for construction of mini tunnels / N.Glebov, A.Vokalyuk, V. Nadtoka. - № 2009129681/03; publ. 10.12.2010, bul. № 34 . 